\title{
Energy Efficiency Design for Secure MISO Cognitive Radio Network Based on a Nonlinear EH Model
}

\author{
Lei $\mathrm{Ni}\left(\mathbb{D},{ }^{1}\right.$ Xinyu $\mathrm{Da}^{2}$ Hang $\mathrm{Hu} \mathbb{D},{ }^{2}$ and Miao $\mathrm{Zhang}^{3}$ \\ ${ }^{1}$ Graduate School, Air Force Engineering University, Xian 710077, China \\ ${ }^{2}$ Information and Navigation College, Air Force Engineering University, Xi'an 710077, China \\ ${ }^{3}$ Department of Electronic Engineering, University of York, York YO10 5DD, UK \\ Correspondence should be addressed to Lei Ni; nileikgd@163.com
}

Received 23 November 2017; Revised 29 March 2018; Accepted 10 April 2018; Published 13 May 2018

Academic Editor: Sergey A. Suslov

Copyright (c) 2018 Lei Ni et al. This is an open access article distributed under the Creative Commons Attribution License, which permits unrestricted use, distribution, and reproduction in any medium, provided the original work is properly cited.

In this work, we investigate the secrecy energy efficiency (SEE) optimization problem for a multiple-input single-output (MISO) cognitive radio (CR) network based on a practical nonlinear energy-harvesting (EH) model. In particular, the energy receiver (ER) is assumed to be a potential eavesdropper due to the open architecture of a CR network with simultaneous wireless information and power transfer (SWIPT), such that the confidential message is prone to be intercepted in wireless communications. The aim of this work is to provide a secure transmit beamforming design while satisfying the minimum secrecy rate target, the minimum $\mathrm{EH}$ requirement, and the maximum interference leakage power to primary user (PU). In addition, we consider that all the channel state information (CSI) is perfectly known at the secondary transmitter (ST). We formulate this beamforming design as a SEE maximization problem; however, the original optimization problem is not convex due to the nonlinear fractional objective function. To solve it, a novel iterative algorithm is proposed to obtain the globally optimal solution of the primal problem by using the nonlinear fractional programming and sequential programming. Finally, numerical simulation results are presented to validate the performance of the proposed scheme.

\section{Introduction}

As a promising approach to simultaneously improving both energy efficiency (EE) and spectrum efficiency (SE) in next generation wireless communication systems [1], the wideband energy-harvesting CR network has attracted significant attention recently. The harvested energy is environmentally friendly as it can greatly reduce carbon emissions and enable the energy-limited sensor node or low-power devices to operate continuously without periodic battery replacements [2]. Energy harvesting from radio frequency (RF) signals is more controllable and reliable than other ambient renewable resources, such as solar and wind; thus it is able to provide relatively stable power to wireless communication networks [3]. Many existing works on energy-harvesting CR network adopted a linear EH model, where it was assumed that the conversed power at the ER linearly increases with the input power [4]. However, in practice, both the harvested energy and the practical power conversion are highly nonlinear; thus, employing the linear EH model to characterize the endto-end wireless energy transmission may lead to resource allocation mismatch.

As is known to us all, RF signals can transfer information jointly with energy via the same carrier, which facilitates SWIPT technique [5-8]. However, due to the inherent nature of SWIPT and the openness of wireless medium, malicious receivers within the cover range can illegitimately access the spectrum bands and analyze the transmission without being detected, which makes the confidential messages transmitted in SWIPT systems vulnerable to eavesdropping [9]. Consequently, how to achieve secure communication while guaranteeing efficient $\mathrm{EH}$ is of significant importance for $\mathrm{CR}$ network. To handle this challenge, an emerging technique termed as PHY layer security has attracted considerable interest in recent years [10-14]. By exploiting the physical properties of wireless channels, PHY layer security has great 
potential to achieve "perfect secrecy" and is identified as a significant complement to traditional cryptographic techniques. To the best of the authors' knowledge, the SEE maximization problem in CR network with SWIPT based on a nonlinear EH model is still in its infancy. Motivated by the aforementioned aspects, we formulate the transmit covariance matrix design problem to maximize the SEE under the constraints of PU's quality-of-service (QoS) requirement, EH requirement, and total transmit power limit for CR network. In addition, the $\mathrm{ER}$ is considered to be a potential eavesdropper and the transmission is susceptible to be intercepted. The original SEE maximization problem is nonconvex and thus difficult to solve directly. To circumvent this issue, the variable substitutions and semidefinite relaxation (SDR) are used, and we reformulate the optimization problem into a tractable form by exploiting an iterative algorithm which combines nonlinear fractional programming $[15,16]$ and difference-ofconcave functions (DC) approaches [17].

The remainder of this paper is organized as follows. In Section 2, the system model is presented. Section 3 presents the SEE maximization problem under the assumption of perfect channel state information (CSI) available at the transmitter, and a novel iterative algorithm is proposed to solve the optimization problem. Section 4 provides simulation results to validate the performance of the proposed algorithms and finally conclusions are addressed in Section 5.

Notations. Vectors and matrices are denoted by lower and upper case boldface letters, respectively. $\mathbb{M}^{N}$ represents the set of Hermitian matrices with $N \times N$ size, and I represents an identity matrix with appropriate size. $\operatorname{Rank}(\mathbf{A})$ denotes the rank of matrix $\mathbf{A}$, and $\operatorname{Tr}(\mathbf{A})$ indicates the trace of matrix $\mathbf{A}$. $(\cdot)^{-1}$ and $(\cdot)^{H}$ stand for the inverse and Hermitian transpose operations, respectively. $\mathbf{A} \succeq 0(\mathbf{A}>0)$ means that the matrix A is a Hermitian positive semidefinite (definite) matrix. The distribution of a circularly symmetric complex Gaussian (CSCG) random variable with mean $\mu$ and variance $\sigma^{2}$ is denoted by $\mathscr{C} \mathscr{N}\left(\mu, \sigma^{2}\right)$, and finally the symbol $\mathbb{E}(\cdot)$ denotes the expectation.

\section{System Model and Problem Statement}

2.1. System Model. As depicted in Figure 1, a downlink MISO energy-harvesting CR network with SWIPT is considered. The ST is equipped with $N_{t}$ antennas while the primary receiver (PR), SR, and ER have all a single antenna. Since the RF signals transmitted by ST and SR carry confidential information, a potential issue might arise as the ER is a passive eavesdropper in this CR network. In this paper, we focus on the worst-case scenario whereby ER can only harvest energy when it does not attempt to decode the confidential message. The channel coefficients between the ST and PR, SR, and ER are denoted by $\mathbf{h}_{p} \in \mathbb{C}^{N_{t} \times 1}, \mathbf{h}_{s} \in \mathbb{C}^{N_{t} \times 1}$, and $\mathbf{h}_{e} \in \mathbb{C}^{N_{t} \times 1}$, respectively. Then, the signals received at $\mathrm{SR}$ and $\mathrm{ER}$ can be written as

$$
\begin{aligned}
& y_{s}=\mathbf{h}_{s}^{H} \mathbf{w} s+n_{s} \\
& y_{e}=\mathbf{h}_{e}^{H} \mathbf{w} s+n_{e},
\end{aligned}
$$

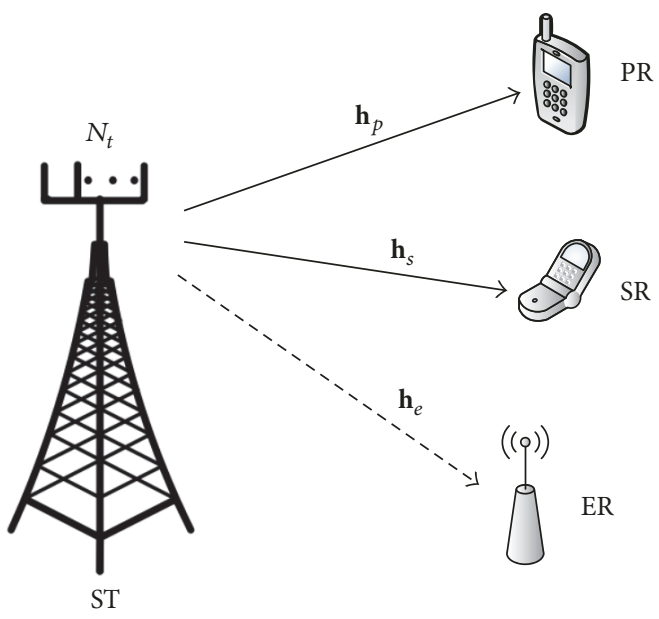

Figure 1: The system model.

where $\mathbf{w} \in \mathbb{C}^{N_{t} \times 1}$ is the corresponding precoding beamforming vector and $s \in \mathbb{C}$ represents the confidential informationbearing symbol intended for SR. Without loss of generality, we assume that $\mathbb{E}\left[|s|^{2}\right]=1 . n_{s} \sim \mathscr{C} \mathscr{N}(0,1)$ and $n_{e} \sim \mathscr{C} \mathscr{N}(0,1)$ denote the additive white Gaussian noise (AWGN) at SR and $\mathrm{ER}$, respectively.

According to the notion of information-theoretic security, the achievable secrecy rate $R_{s}$ of SR can be expressed as

$$
R_{s}=C_{s}-C_{e},
$$

where $C_{s}$ and $C_{e}$ are given by

$$
\begin{aligned}
& C_{s}=\log _{2}\left(1+\mathbf{h}_{s}^{H} \mathbf{W} \mathbf{h}_{s}\right) \\
& C_{e}=\log _{2}\left(1+\mathbf{h}_{e}^{H} \mathbf{W} \mathbf{h}_{e}\right),
\end{aligned}
$$

where $\mathbf{W}=\mathbf{w} \mathbf{w}^{H}$ is the transmit covariance matrix. $\operatorname{Tr}(\mathbf{W}) \leqslant$ $P$, and $P$ denotes the maximum transmit power at the transmitter.

Following the similar power consumption model utilized in [14], the total power consumption at ST can be written as

$$
P_{t}=\frac{\operatorname{Tr}(\mathbf{W})}{\xi}+P_{c},
$$

where $\xi \in(0,1]$ is the power amplifier efficiency and $P_{c}$ is the average energy consumption of the transmitter circuits. $\xi$ is assumed to be one without loss of generality in this paper; that is, $P_{t}=\operatorname{Tr}(\mathbf{W})+P_{c}$. The SEE is defined as the ratio between the achievable secrecy rate and the total power consumption, which can be expressed as

$$
\eta=\frac{R_{s}}{P_{t}}=\frac{\log _{2}\left(1+\mathbf{h}_{s}^{H} \mathbf{W} \mathbf{h}_{s}\right)-\log _{2}\left(1+\mathbf{h}_{e}^{H} \mathbf{W} \mathbf{h}_{e}\right)}{\operatorname{Tr}(\mathbf{W})+P_{c}} .
$$

2.2. Energy-Harvesting Model. In the existing literature on the SWIPT, the power converted by the energy-harvesting receiver is typically modeled as an overly simplistic linear $\mathrm{EH}$ model, which can be defined as

$$
\Phi_{\mathrm{ER}}^{\text {linear }}=\eta_{\text {linear }} P_{\mathrm{ER}}, \quad P_{\mathrm{ER}}=\operatorname{Tr}\left(\mathbf{w} \mathbf{w}^{H} \mathbf{h}_{e} \mathbf{h}_{e}^{H}\right),
$$


where $\eta_{\text {linear }} \in[0,1]$ is a fixed constant that indicates the power conversion efficiency, $\Phi_{\mathrm{ER}}^{\text {linear }}$ is the power conversed at the ER, and $P_{\mathrm{ER}}$ is the received RF power at the ER.

In the linear $\mathrm{EH}$ model, the conversion efficiency is independent of the received RF signals. However, in practice, the power conversion efficiency is greatly relative to the input power level and the $\mathrm{EH}$ circuits generally result in a nonlinear end-to-end wireless power transfer. According to the practical nonlinear EH model given by [18], the harvested energy $\Phi_{\mathrm{ER}}$ at the ER is typically modeled as

$$
\Phi_{\mathrm{ER}}\left(P_{\mathrm{ER}}\right)=\frac{\psi_{\mathrm{ER}}-M \Psi_{\mathrm{ER}}}{1-\Psi_{\mathrm{ER}}},
$$

where $\psi_{\mathrm{ER}}=M /\left(1+e^{-a\left(P_{\mathrm{ER}}-b\right)}\right), \Psi_{\mathrm{ER}}=1 /\left(1+e^{a b}\right)$, and $M$ is a constant, denoting the maximum harvested energy of ER when the actual EH circuit reaches saturation. $a$ and $b$ are constants that indicate the joint effects of various nonlinear phenomena caused by hardware limitation. In practice, one can readily determine parameters $M, a$, and $b$ by the standard curve fitting method.

\section{Problem Formulation}

In this section, our objective is to maximize the SEE of energy-harvesting CR network with SWIPT while satisfying the PR's QoS requirement and EH constraint. Thus, the SEE maximization problem can be mathematically formulated as follows:

$$
\begin{array}{ll}
\max _{\mathbf{W}} \quad \eta=\frac{R_{s}}{P_{t}} & \\
& =\frac{\log _{2}\left(1+\mathbf{h}_{s}^{H} \mathbf{W} \mathbf{h}_{s}\right)-\log _{2}\left(1+\mathbf{h}_{e}^{H} \mathbf{W} \mathbf{h}_{e}\right)}{\operatorname{Tr}(\mathbf{W})+P_{c}} \\
\text { s.t. } \quad \mathrm{C} 1: R_{s} & \\
& =\log _{2}\left(1+\mathbf{h}_{s}^{H} \mathbf{W h}_{s}\right)-\log _{2}\left(1+\mathbf{h}_{e}^{H} \mathbf{W h}_{e}\right) \\
& \geqslant R_{d}, \\
& \mathrm{C} 2: \Phi_{\mathrm{ER}} \geqslant E_{s}, \\
& \mathrm{C} 3: \mathbf{h}_{p}^{H} \mathbf{W} \mathbf{h}_{p} \leqslant P_{p}, \\
& \mathrm{C} 4: \operatorname{Tr}(\mathbf{W}) \leqslant P, \\
& \mathrm{C} 5: \mathbf{W} \geq 0 .
\end{array}
$$

In (8a)-(8f), the constraint $\mathrm{Cl}$ is given to guarantee the security of ST in the secondary network. C2 guarantees that energy harvested by the ER is no less than $E_{s}$ when it does not attempt to eavesdrop the confidential information. The interference power constraint $\mathrm{C} 3$ is imposed in order to protect the QoS of PU and C4 denotes the transmit power constraint of the ST.

Due to the fractional objective function as well as the harvested energy constraint $\mathrm{C} 2$, the problem in $(8 \mathrm{a})-(8 \mathrm{f})$ is not convex and very difficult to solve. In what follows, we first handle the nonconvex constraint and then propose an iterative method to find the optimal solution to the above complex problem. The nonlinear EH function (7) for ER can be reformulate as

$$
P_{\mathrm{ER}}\left(\Phi_{\mathrm{ER}}\right)=b-\frac{1}{a} \ln \left(\frac{e^{a b}\left(M-\Phi_{\mathrm{ER}}\right)}{e^{a b} \Phi_{\mathrm{ER}}+M}\right) .
$$

By defining $E_{0} \triangleq P_{\mathrm{ER}}\left(\Phi_{\mathrm{ER}}\right), \mathbf{X} \triangleq \mathbf{x} \mathbf{x}^{H}, \mathbf{Y} \triangleq \mathbf{y} \mathbf{y}^{H}$, according to the fact that $\mathbf{y} \mathbf{X y}{ }^{H}=\operatorname{Tr}\left(\mathbf{X} \mathbf{y} \mathbf{y}^{H}\right)=\operatorname{Tr}(\mathbf{X Y})$, where $\mathbf{x}$ and $\mathbf{y}$ are vectors, the constraint $\mathrm{C} 2$ can be transformed into

$$
\operatorname{Tr}\left(\mathbf{W H}_{e}\right) \geqslant E_{0} .
$$

Then, problem (8a)-(8f) can be reformulated to be problem (11a)-(11g) equivalently:

$$
\begin{array}{ll}
\max _{\mathbf{W}} \quad & \eta=\frac{R_{s}}{P_{t}} \\
& =\frac{\log _{2}\left(1+\mathbf{h}_{s}^{H} \mathbf{W} \mathbf{h}_{s}\right)-\log _{2}\left(1+\mathbf{h}_{e}^{H} \mathbf{W} \mathbf{h}_{e}\right)}{\operatorname{Tr}(\mathbf{W})+P_{c}}, \\
\text { s.t. } \quad \mathrm{C} 1: 1+\mathbf{h}_{s}^{H} \mathbf{W h}_{s} \geqslant 2^{R_{d}}\left(1+\mathbf{h}_{e}^{H} \mathbf{W} \mathbf{h}_{e}\right), \\
\\
\mathrm{C} 2: \operatorname{Tr}\left(\mathbf{W H}_{e}\right) \geqslant E_{0}, \\
\mathrm{C} 3: \mathbf{h}_{p}^{H} \mathbf{W} \mathbf{h}_{p} \leqslant P_{p}, \\
\mathrm{C} 4: \operatorname{Tr}(\mathbf{W}) \leqslant P, \\
\mathrm{C} 5: \mathbf{W} \geq 0, \\
\mathrm{C} 6: \operatorname{Rank}(\mathbf{W}) \leqslant 1 .
\end{array}
$$

As a result, we handle it with SDR by dropping the rank-one matrix C6. Specifically, we relax constraint C6 in (11a)-(11g) by removing constraint $\operatorname{Rank}\left(\mathbf{W}^{*}\right) \leqslant 1$ from the problem. Nevertheless, problem (11a)-(11g) is still a nonconvex problem due to the fractional objective function. Thereby, it cannot be solved directly by using traditional method [19]. To tackle this difficulty, we convert this problem into a convex one through nonlinear fractional and sequential convex programming in the following subsections.

3.1. Nonlinear Fractional Programming. The optimization problem in (11a)-(11g) is known as a fractional programming problem with nonlinear as well as linear terms in the numerator and denominator. To solve it, the original problem is transformed into a parameter problem with respect to $\lambda>$ 0 , given as 


$$
\begin{aligned}
& \mathscr{F}(\lambda)=\max _{\mathbf{W}} \quad\left[R_{s}-\lambda P_{t}\right]=\max _{\mathbf{W}}\left\{\log _{2}\left(1+\mathbf{h}_{s}^{H} \mathbf{W h}_{s}\right)-\log _{2}\left(1+\mathbf{h}_{e}^{H} \mathbf{W} \mathbf{h}_{e}\right)-\lambda\left[\operatorname{Tr}(\mathbf{W})+P_{c}\right]\right\} \\
& \text { s.t. } \quad \mathrm{C} 1: 1+\mathbf{h}_{s}^{H} \mathbf{W} \mathbf{h}_{s} \geqslant 2^{R_{d}}\left(1+\mathbf{h}_{e}^{H} \mathbf{W} \mathbf{h}_{e}\right), \\
& \mathrm{C} 2: \operatorname{Tr}\left(\mathbf{W} \mathbf{H}_{e}\right) \geqslant E_{0}, \\
& \mathrm{C} 3: \mathbf{h}_{p}^{H} \mathbf{W} \mathbf{h}_{p} \leqslant P_{p}, \\
& \mathrm{C} 4: \operatorname{Tr}(\mathbf{W}) \leqslant P, \\
& \mathrm{C} 5: \mathbf{W} \succeq 0 .
\end{aligned}
$$

Proposition 1. Denote that $\lambda^{*}$ is the unique zero solution to $\mathscr{F}(\lambda)$; then $\mathscr{F}\left(\lambda^{*}\right)$ has the same optimal solution as the objective function in (11a)-(11g), and the maximum SEE can be derived when the following condition is satisfied:

$$
\mathscr{F}\left(\lambda^{*}\right)=R_{s}^{*}-\lambda^{*} P_{t}^{*}=0,
$$

where $R_{s}^{*}$ and $P_{t}^{*}$ represent the optimal secrecy rate and power consumption, respectively.

Proof. Please refer to [15].

From this proposition, the optimal $\left(R_{s}, P_{t}\right)$ of problem (12a)-(12f) can be obtained by seeking the unique zero solution of $\mathscr{F}(\lambda)$. Hence, our strategy is to optimize (12a)-(12f) with a given $\lambda$ and iteratively seek $\lambda^{*}$ based on Dinkelbach's method.

3.2. Sequential Programming. As is known to us all, DC programming is an optimization method to solve nonconvex problems. Particularly, this technique can be applied to an optimization problem when the objective function is in a difference-of-concave form. Since the context of problem (12a)-(12f) falls into this category, DC programming can be utilized to solve this issue. Sequential programming can tackle the DC problems but is not limited to DC programming and indeed it has been used in conjunction with fractional optimization in previous works [11, 12]. The basic idea of our sequential method is to locally linearize the nonconcave functions in (12a)-(12f) at some feasible point $\mathbf{W}^{k}$ and then iteratively solve the linearized problem; that is,

$$
\begin{aligned}
\max _{\mathbf{W}} & \left\{\log _{2}\left(1+\mathbf{h}_{s}^{H} \mathbf{W} \mathbf{h}_{s}\right)-f\left(\mathbf{W}, \mathbf{W}^{k}\right)-\lambda_{i}\left[\operatorname{Tr}(\mathbf{W})+P_{c}\right]\right\} \\
\text { s.t. } & (12 \mathrm{~b}),(12 \mathrm{c}),(12 \mathrm{~d}),(12 \mathrm{e}),(12 \mathrm{f}),
\end{aligned}
$$

where we define the function $f\left(\mathbf{W}, \mathbf{W}^{k}\right)=\log _{2}(1+$ $\left.\mathbf{h}_{e}^{H} \mathbf{W}^{k} \mathbf{h}_{e}\right)+\mathbf{h}_{e}^{H}\left(\mathbf{W}-\mathbf{W}^{k}\right) \mathbf{h}_{e} /\left(1+\mathbf{h}_{e}^{H} \mathbf{W} \mathbf{h}_{e}\right) \ln 2$ to approximate the second term of the objective function in (12a)-(12f).

Thus, the original fractional problem to be solved in each iteration of the sequential programming takes the form shown in (14) [11]. It is easy to prove that the approximated problem in (14) is convex with respect to $\mathbf{W}$, and the optimal $\mathbf{W}^{*}$ can be iteratively obtained by solving problem (14) based on updating $\mathbf{W}^{k}$. According to the above analysis, an algorithm characterized by fractional programming and sequential convex optimization is developed, which solves the SEE maximization problem by two-layer embedded iterations. The process of proposed iterative algorithm is stated as follows:

(1) Input settings: the error tolerances $\varepsilon>0, \zeta>0$;

(2) Initialization $i=0$, choose an initial value $\lambda_{i}$ such that $\mathscr{F}\left(\lambda_{i}\right)>0$;

(3) Repeat

(4) For the given $\lambda_{i}$, search the optimal $R_{s}^{i}$ and $P_{t}^{i}$ of (12a)-(12f);

(5) Initial $k=0, \mathbf{W}^{k}=0$ and $\eta^{i, k}=0$;

(6) Repeat

(7) Solve the problem (14) with $\lambda=\lambda_{i}$ via interiorpoint method and obtain $\mathbf{W}^{k+1}$;

(8) Compute $\eta^{i, k+1}=\log _{2}\left(1+\mathbf{h}_{s}^{H} \mathbf{W}^{k+1} \mathbf{h}_{s}\right)-\log _{2}(1+$ $\left.\mathbf{h}_{e}^{H} \mathbf{W}^{k+1} \mathbf{h}_{e}\right)-\lambda_{i}\left[\operatorname{Tr}\left(\mathbf{W}^{k+1}\right)+P_{c}\right] ;$

(9) $\Delta \eta=\eta^{i, k+1}-\eta^{i, k}$;

(10) Update $k=k+1$;

(11) Until $|\Delta \eta|<\zeta$

(12) $\operatorname{Return} R_{s}^{i}=\log _{2}\left(1+\mathbf{h}_{s}^{H} \mathbf{W}^{k} \mathbf{h}_{s}\right)-\log _{2}\left(1+\mathbf{h}_{e}^{H} \mathbf{W}^{k} \mathbf{h}_{e}\right)$ and $P_{t}^{i}=\operatorname{Tr}\left(\mathbf{W}^{k}\right)+P_{c}$ for updating $\lambda_{i}$;

(13) Update $\lambda_{i+1}=R_{s}^{i} / P_{t}^{i}$ to obtain $\lambda_{i+1}$;

(14) $i=i+1$;

(15) Until $\Delta F=\left|R_{s}^{i}-\lambda_{i} P_{t}^{i}\right| \leqslant \varepsilon$ satisfied;

(16) Return $\lambda^{*}=\lambda_{i}, P_{t}^{*}=P_{t}^{i-1}, R_{s}^{*}=R_{s}^{i-1}$.

It is worth noting that only when $\operatorname{Rank}\left(\mathbf{W}^{*}\right)=1$, the optimal solution, that is, $\mathbf{w}^{*}$ is recovered by the eigenvalue decomposition of $\mathbf{W}^{*}$. Otherwise, the approximate optimal solution is obtained by using the largest eigenvalue of $\mathbf{W}^{*}$ [20]. Provided that problem (1la)-(11g) is feasible, we will find that the optimal $\mathbf{W}^{*}$ will be always rank-one. In other words, the SDR is tight and the global optimal solution to the primal problem can be guaranteed; besides, the corresponding SEE can be attained via single-stream transmit beamforming. 




(a)

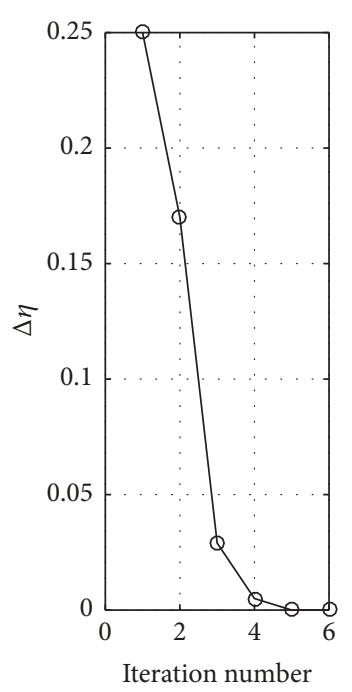

(b)

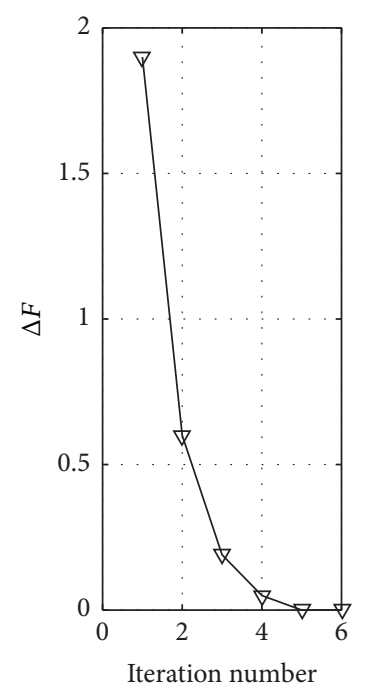

(c)

FIGURE 2: Convergence results of the proposed algorithm.

\section{Numerical Results}

In this section, we provide numerical simulation results to testify the availability of our proposed scheme. In our examples, the ST is equipped with three antennas, while the $\mathrm{PR}, \mathrm{SR}$, and ER each use a single antenna. The maximum interference leakage to the $\mathrm{PU}$ is assumed to be $0 \mathrm{~dB}$, and the energy conversion ratio is assumed to be 0.5 . Besides, all the channel coefficients are generated by CSCG with zero mean and unit variance. Regarding the nonlinear $\mathrm{EH}$ model parameters, we adopt $M=28 \mathrm{~mW}, a=6400$, and $b=0.003$. In addition, the convergence tolerances $\varepsilon$ and $\zeta$ are set to be $10^{-3}$.

Figure 2 evaluates the convergence behavior of the proposed algorithm. Here, the maximum transmit power $P$ and the energy-harvesting requirement $E_{s}$ are assumed to be $20 \mathrm{~dB}$ and $0 \mathrm{~dB}$, respectively. Figure 2 (a) illustrates the convergence of achieved SEE for the target secrecy rate $R_{d}=$ $0.5 \mathrm{bps} / \mathrm{Hz}$. Moreover, the convergence of parameters $\Delta \eta$ and $\Delta F$, which describe the termination of the iterative processes, is depicted in Figures 2(b) and 2(c), respectively. From the simulation results, we can see that the maximum SEE and the convergence can be achieved with a limited number of iterations.

Figure 3 depicts the achieved SEE versus the target secrecy rate for different transmit power consumption and $\mathrm{EH}$ requirements. As can be observed, the optimal SEE decreases as the target secrecy rate increases. Note that the zero SEE means that the optimization problem is not feasible. If the problem is feasible with a given target secrecy rate constraint, we notice that the SEE can achieve a better performance with a larger transmit power consumption and smaller $\mathrm{EH}$ requirement. In addition, increasing the total transmit power is able to achieve a higher target secrecy rate.

Figure 4 shows the average SEE of the proposed scheme versus the maximum transmit power $P$. The target secrecy



FIGURE 3: Achieved SEE with different target secrecy rates.

rate and the energy-harvesting requirement $E_{s}$ are assumed to be $0.5 \mathrm{bps} / \mathrm{Hz}$ and $0 \mathrm{~dB}$, respectively. Here, the results of the secrecy rate maximization (SRM) [21] and the energy efficiency maximization (EEM) [22] scheme are also provided for comparison, and it is observed that the proposed SEE maximization scheme achieves a similar performance to that of the SRM scheme when $P \leqslant 32 \mathrm{dBm}$, since both schemes use all of the transmit power $P$ to obtain the maximum SEE. However, the performance of SRM scheme is degraded drastically as $P$ increases, while the SEE maximization scheme remains the same. This can be explained as follows. The performance gain of our proposed scheme is kept because it ceases allocating more transmit power to 


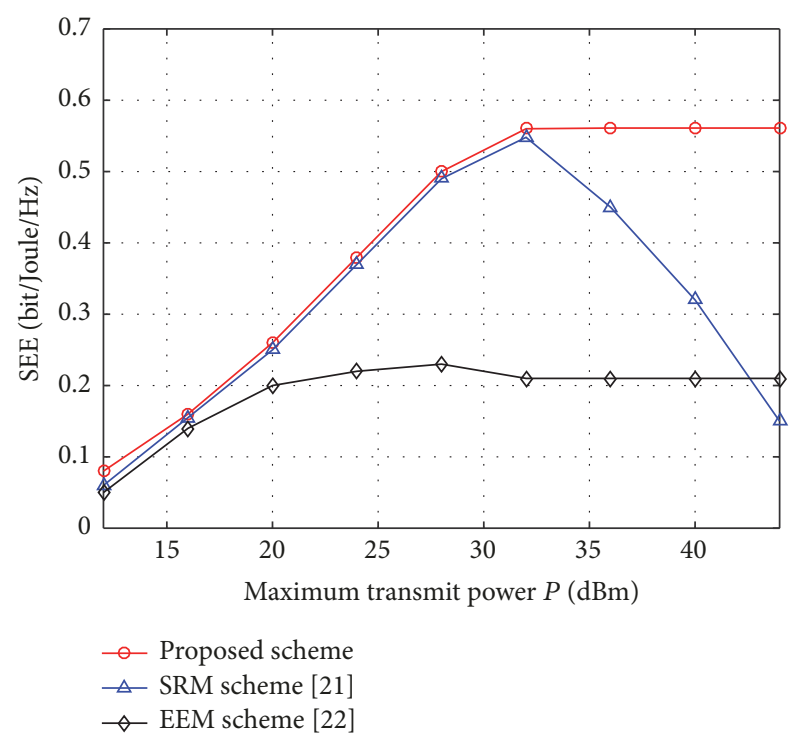

FIgURE 4: Achieved SEE versus the maximum transmit power $P$.

avoid sacrificing the SEE; besides, exceedingly large transmit power can cause saturation in EH circuits. However, the SRM scheme still increases transmit power to obtain higher secrecy rate. Meanwhile, both of the proposed schemes significantly outperform the EEM scheme which focuses only on the EE and ignores the existence of the potential eavesdropper.

\section{Conclusions}

In this paper, we study the secure beamforming design for SWIPT based on a nonlinear EH model considering a potential eavesdropper. In particular, the transmit covariance matrix is designed to maximize the SEE while meeting the $\mathrm{EH}$ requirement and interference power constraint. Since the SEE maximization problem is not convex and thus difficult to solve directly, we convert the original problem into a convex one by exploiting nonlinear fractional and sequential programming. Simulation results are provided to validate the performance of our proposed scheme.

\section{Conflicts of Interest}

The authors declare that there are no conflicts of interest regarding the publication of this paper.

\section{Acknowledgments}

This work is supported in part by the Natural Science Foundation of China under Grant 61571460 and Grant 60972042 and in part by the National Postdoctoral Program for Innovative Talents under Grant BX201700108.

\section{References}

[1] H. Hu, Y.-C. Liang, H. Zhang, and B.-H. Soong, "Cognitive radio with self-power recycling," IEEE Transactions on Vehicular Technology, vol. 66, no. 7, pp. 6201-6214, 2017.
[2] Z. Hadzi-Velkov, I. Nikoloska, G. K. Karagiannidis, and T. Q. Duong, "Wireless networks with energy harvesting and power transfer: joint power and time allocation," IEEE Signal Processing Letters, vol. 23, no. 1, pp. 50-54, 2016.

[3] S. Khoshabi Nobar, K. Adli Mehr, and J. Musevi Niya, "RFpowered green cognitive radio networks: architecture and performance analysis," IEEE Communications Letters, vol. 20, no. 2, pp. 296-299, 2016.

[4] K. Xiong, B. Wang, and K. J. R. Liu, "Rate-Energy Region of SWIPT for MIMO Broadcasting under Nonlinear Energy Harvesting Model," IEEE Communications Letters, vol. 16, no. 8, pp. 5147-5161, 2017.

[5] G. Pan, H. Lei, Y. Yuan, and Z. Ding, "Performance Analysis and Optimization for SWIPT Wireless Sensor Networks," IEEE Transactions on Communications, vol. 65, no. 5, pp. 2291-2302, 2017.

[6] W. Mei, Z. Chen, and J. Fang, "Secrecy Capacity Region Maximization in Gaussian MISO Channels with Integrated Services," IEEE Signal Processing Letters, vol. 23, no. 8, pp. 11461150, 2016.

[7] W. Mei, Z. Chen, and J. Fang, "Artificial Noise Aided Energy Efficiency Optimization in MIMOME System with SWIPT," IEEE Communications Letters, vol. 21, no. 8, pp. 1795-1798, 2017.

[8] H. Niu, B. Zhang, D. Guo, and Y. Huang, "Joint Robust Design for Secure AF Relay Networks with SWIPT," IEEE Access, vol. 5, pp. 9369-9377, 2017.

[9] Z. Chu, Z. Zhu, and J. Hussein, "Robust optimization for ANaided transmission and power splitting for secure MISO SWIPT system," IEEE Communications Letters, vol. 20, no. 8, pp. 15711574, 2016.

[10] H. Yu, D. Wang, G. Pan, R. Shi, J. Zhang, and Y. Chen, "On Outage of WPC System with Relay Selection over Nakagami-m Fading Channels," IEEE Transactions on Vehicular Technology, vol. 66, no. 9, pp. 8590-8594, 2017.

[11] A. Zappone, P.-H. Lin, and E. Jorswieck, "Energy Efficiency of Confidential Multi-Antenna Systems with Artificial Noise and Statistical CSI," IEEE Journal of Selected Topics in Signal Processing, vol. 10, no. 8, pp. 1462-1477, 2016.

[12] A. Zappone, P.-H. Lin, and E. Jorswieck, "Optimal EnergyEfficient Design of Confidential Multiple-Antenna Systems," IEEE Transactions on Information Forensics and Security, 2017.

[13] E. Boshkovska, D. W. K. Ng, N. Zlatanov, A. Koelpin, and R. Schober, "Robust Resource Allocation for MIMO Wireless Powered Communication Networks Based on a Non-Linear EH Model," IEEE Transactions on Communications, vol. 65, no. 5, pp. 1984-1999, 2017.

[14] J. Xu and L. Qiu, "Energy efficiency optimization for MIMO broadcast channels," IEEE Transactions on Wireless Communications, vol. 12, no. 2, pp. 690-701, 2013.

[15] W. Dinkelbach, "On Nonlinear Fractional Programming," Management Science, vol. 13, no. 7, pp. 492-498, 1967.

[16] A. Zappone and E. Jorswieck, "Energy efficiency in wireless networks via fractional programming theory," Foundations and Trends in Communication and Information Theory, vol. 11, no. 3-4, pp. 185-396, 2015.

[17] T. P. Dinh and H. A. L. Thi, Recent advances in DC programming and DCA, Transactions on Computational Intelligence XIII, 2014.

[18] E. Boshkovska, D. W. K. Ng, N. Zlatanov, and R. Schober, "Practical non-linear energy harvesting model and resource allocation for SWIPT systems," IEEE Communications Letters, vol. 19, no. 12, pp. 2082-2085, 2015. 
[19] S. Boyd and L. Vandenberghe, Convex Optimization, Cambridge University Press, 2004.

[20] R. Jiang, K. Xiong, P. Fan, Y. Zhang, and Z. Zhong, "Optimal Design of SWIPT Systems with Multiple Heterogeneous Users under Non-linear Energy Harvesting Model," IEEE Access, vol. 5, pp. 11479-11489, 2017.

[21] J. Mao, G. Xie, J. Gao, and Y. Liu, "Energy efficiency optimization for cognitive radio MIMO broadcast channels," IEEE Communications Letters, vol. 17, no. 2, pp. 337-340, 2013.

[22] F. Haider, C.-X. Wang, H. Haas, E. Hepsaydir, X. Ge, and D. Yuan, "Spectral and energy efficiency analysis for cognitive radio networks," IEEE Transactions on Wireless Communications, vol. 14, no. 6, pp. 2969-2980, 2015. 


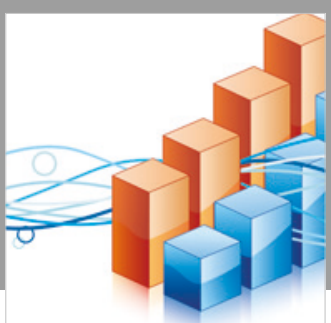

Advances in

Operations Research

\section{-n-m}
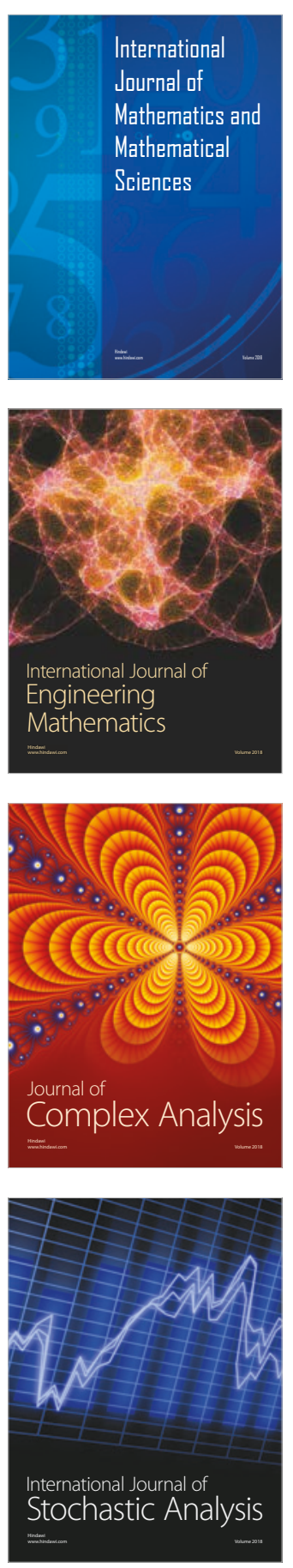
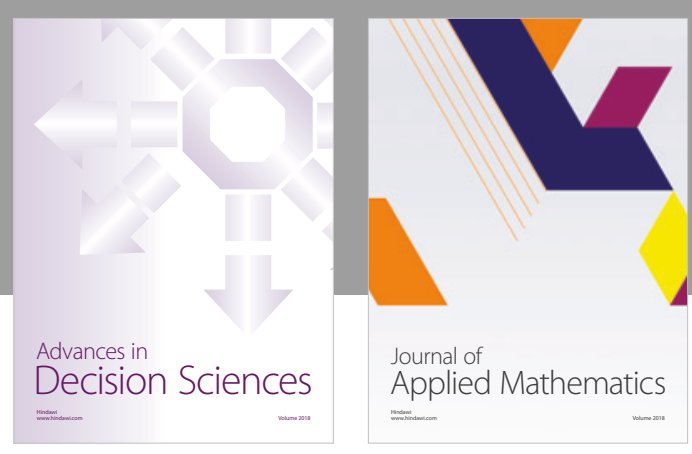

Journal of

Applied Mathematics
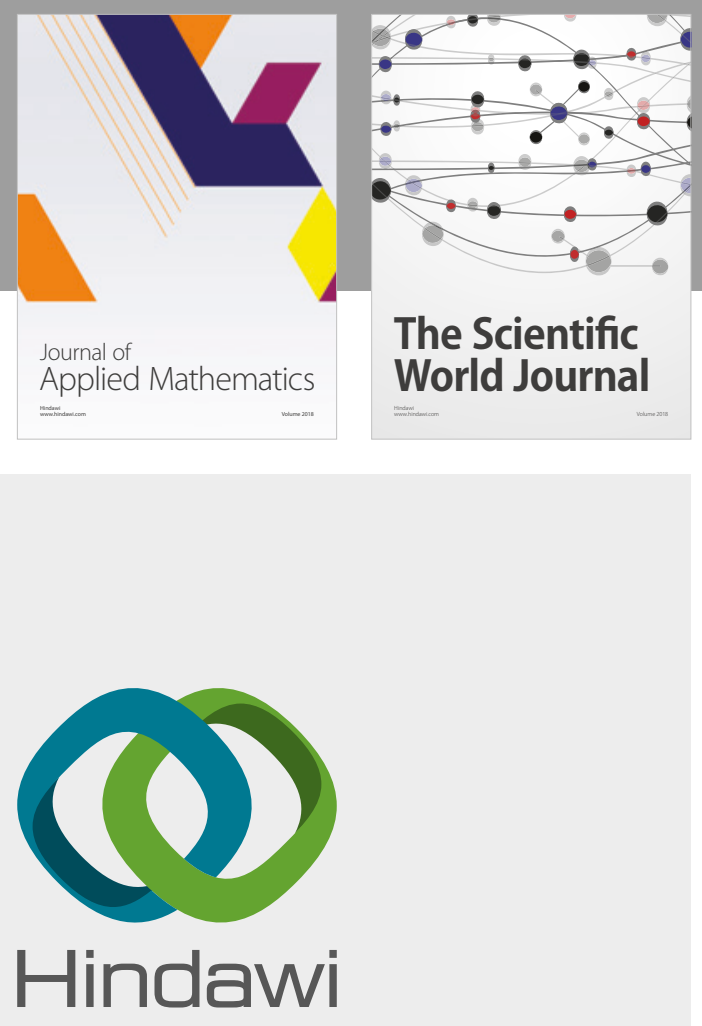

Submit your manuscripts at

www.hindawi.com

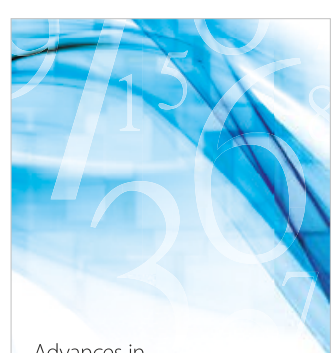

Advances in
Numerical Analysis
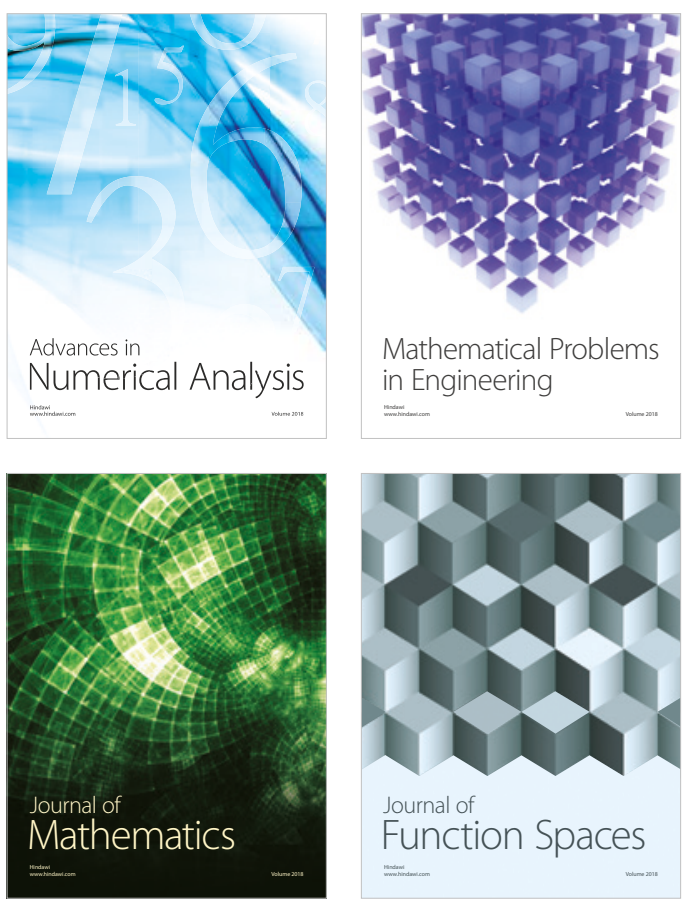

Mathematical Problems in Engineering

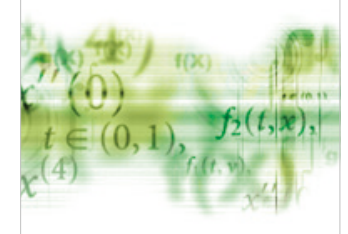

International Journal of

Differential Equations

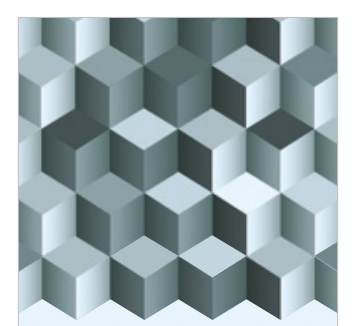

Journal of

Function Spaces



The Scientific

World Journal

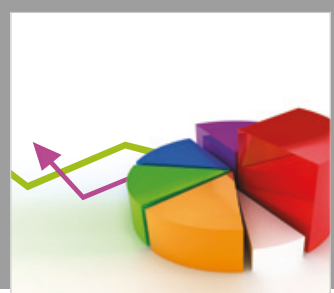

Journal of

Probability and Statistics
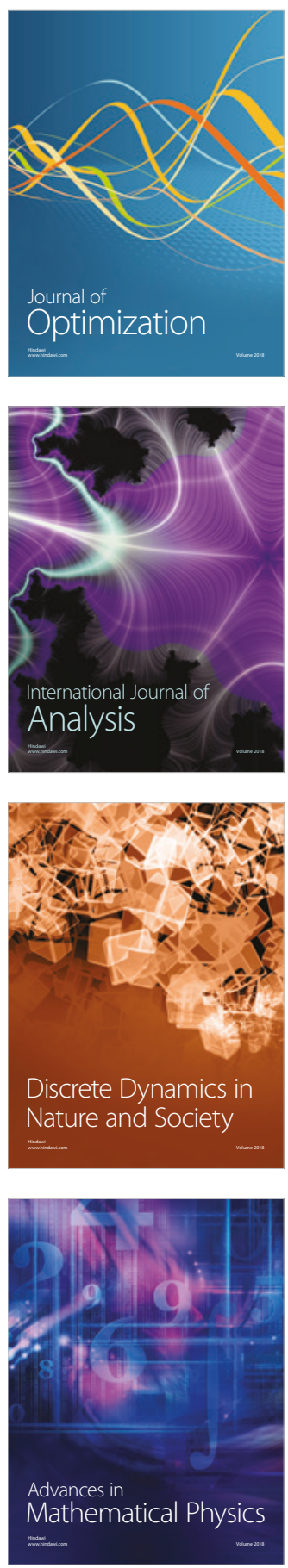